\title{
Síndrome de reacción a fármacos con eosinofilia y síntomas sistémicos inducido por carbamazepina de liberación prolongada: reporte de un caso
}

Elman Rolando Gamarra Osorio* 1,2,a; Deborah Ximena Arzani Lezcano 1,2,b; Olga Mercedes Viviana Burgos Garcia 1,3,c

\section{RESUMEN}

El síndrome de reacción a fármacos con eosinofilia y síntomas sistémicos es una reacción de hipersensibilidad a fármacos poco común, pero con una alta mortalidad, por ello se requiere un diagnóstico precoz y un manejo oportuno. Presentamos el caso de una mujer de 32 años con diagnóstico de epilepsia y trastorno esquizofreniforme orgánico, secundarios a encefalitis viral, y que ha recibido tratamiento con múltiples fármacos. Tres semanas después de añadir carbamazepina de liberación prolongada a su terapia habitual, la paciente presentó una erupción cutánea difusa tipo habón, edema facial, fiebre, linfadenopatía, leucocitosis con eosinofilia y elevación de las transaminasas. La administración de la carbamazepina fue suspendida, se administró antihistamínicos y glucocorticoides por vía oral, y la paciente mejoró.

Palabras clave: Síndrome de hipersensibilidad a medicamentos; Carbamazepina; Exantema; Eosinofilia (Fuente: DeCS BIREME).

\section{Drug reaction with eosinophilia and systemic symptoms syndrome caused by extended-release carbamazepine: a case report}

\section{ABSTRACT}

The drug reaction with eosinophilia and systemic symptoms (DRESS) syndrome is a rare but highly lethal drug hypersensitivity reaction. Thus, it requires an early diagnosis and timely management. We present the case of a 32-year-old female patient with a diagnosis of epilepsy and organic schizophreniform disorder, secondary to viral encephalitis, who was treated with multiple drugs. Three weeks after the addition of extended-release carbamazepine to her usual therapy, the patient presented a diffuse welt-type skin rash, facial edema, fever, lymphadenopathy, leukocytosis with eosinophilia and elevated transaminases. Carbamazepine administration was discontinued, antihistamines and glucocorticoids were administered orally, and the patient showed a remarkable improvement.

Keywords: Drug hypersensitivity syndrome; Carbamazepine; Exanthema; Eosinophilia (Source: MeSH NLM).

1 Hospital Víctor Lazarte Echegaray. Trujillo, Perú.

2 Universidad Nacional de Trujillo. Trujillo, Perú.

3 Universidad Privada Antenor Orrego. Trujillo, Perú.

a Médico Internista.

b Interna de Medicina.

c Médico Residente de segundo año de Anatomía Patológica.

* Autor corresponsal. 


\section{INTRODUCCIÓN}

El síndrome de reacción a fármacos con eosinofilia y síntomas sistémicos (DRESS, del inglés Drug reaction with eosinophilia and systemic symptoms) es una reacción de hipersensibilidad a fármacos (1), poco común, con una incidencia que varía de $1 / 1000$ a $1 / 10000^{(2,3)}$, pero con una tasa de mortalidad aproximada del $10 \%{ }^{(3,4)}$. Los antibióticos, antivirales y, sobre todo, los anticonvulsivantes se asocian con el síndrome DRESS ${ }^{(5-8)}$, que aparece entre la segunda y la sexta semana después de la exposición al fármaco, aunque puede ocurrir hasta dos o tres meses más tarde ${ }^{(7-12)}$, lo que es menos frecuente. En la patogénesis del síndrome DRESS intervienen los metabolitos tóxicos sintetizados a partir de los fármacos ingeridos, la susceptibilidad genética asociada al antígeno leucocitario humano (HLA), así como la reactivación de los virus herpes humanos $(\mathrm{VHH})^{(5-12)}$.

Las manifestaciones clínicas incluyen erupción cutánea, alteraciones hematológicas y daño de órganos (principalmente hígado, riñón o pulmón), que deben remitir tras la suspensión del fármaco causal, por lo que las medidas terapéuticas más importantes son la suspensión del posible fármaco junto al soporte de los daños sistémicos agudos ${ }^{(5-12)}$.

Los anticonvulsivantes aromáticos como carbamazepina, fenitoína o fenobarbital son los fármacos que se relacionan con mayor frecuencia al síndrome DRESS ${ }^{(5-13)}$. La enzima citocromo 450 metaboliza estas sustancias y produce óxidos de areno que luego son degradados por la enzima epóxido hidroxilasa, por lo que el déficit o las mutaciones en los genes reguladores de estas enzimas ocasionan la acumulación de metabolitos tóxicos que producen una reacción de hipersensibilidad ${ }^{(2,7-10)}$, debido a la activación de interleucina 5 (IL-5) y de eosinófilos que desencadenan la respuesta inflamatoria ${ }^{(7)}$. Otros mecanismos implicados son la reactivación de los $\mathrm{VHH}$, especialmente del $\mathrm{VHH}-6^{(6-12,14)}$, así como la presencia de alelos específicos de los genes HLA, como el alelo HLA-A* 31: 01, que predispone a desarrollar reacciones de hipersensibilidad inducidas por carbamazepina ${ }^{(15)}$.

Presentamos un caso de síndrome DRESS inducido por carbamazepina de liberación prolongada en una paciente epiléptica tratada con múltiples fármacos.

\section{CASO CLÍNICO}

Mujer de 32 años con epilepsia y trastorno esquizofreniforme orgánico secundarios a encefalitis viral ocurrida hace 10 años. Recibe un tratamiento regular con topiramato, clobazam, ácido valproico, levetiracetam, risperidona y biperideno desde hace 4 años. Tres semanas antes del inicio del cuadro, carbamazepina de liberación prolongada (1 tableta de $400 \mathrm{mg}$ cada 12 horas por vía oral). Además, le prescribieron fenitoína hace 9 días por una crisis epiléptica que la llevó a emergencia, y fue dada de alta con la medicación habitual previa. Sin historia previa de reacciones alérgicas. Acude a emergencia por presentar fiebre y erupción cutánea difusa. Al examen físico: regular estado general, presión arterial: $100 / 60 \mathrm{mmHg}$, frecuencia cardiaca de 72 latidos por minuto, frecuencia respiratoria de 18 respiraciones por minuto y temperatura de $39^{\circ} \mathrm{C}$. Presentaba lesiones eritematosas, pruriginosas, tipo habón, difusas en todo el cuerpo y edema palpebral. El examen auxiliar más resaltante es el de transaminasas (Tabla 1). Inician tratamiento con hidrocortisona y clorfenamina y se decide hospitalizar a la paciente. En el cuarto día de la hospitalización el edema ha aumentado en la cara y el cuello. Además, se observan múltiples máculas eritematosas en tronco, abdomen y extremidades superiores e inferiores (Figura 1), también adenomegalia inguinal izquierda y fiebre. Se plantea el diagnóstico de síndrome DRESS y se suspende el tratamiento con carbamazepina. Luego se toma una biopsia de piel (Figuras 3 y 4). Al quinto día aparece un eritema conjuntival y se observa una marcada leucocitosis con eosinofilia (Tabla 1). La ecografía abdominal muestra un parénquima hepático homogéneo, ecogenicidad conservada y vías biliares intrahepáticas no dilatadas. En la biopsia de piel se encuentran epidermis con hipogranulosis, disqueratosis, exocitosis de linfocitos y microvacualización de la membrana basal, dermis papilar con leve infiltrado linfocitario perivascular, polimorfonuclear y escaso infiltrado eosinofílico; y en la inmunohistoquímica, CD3, CD4 y CD8 positivos (Figuras 3 y 4). Al octavo día se suspendió el tratamiento con la hidrocortisona endovenosa y se inició la terapia con prednisona y antihistamínicos por vía oral. Las lesiones eritematosas descamativas en cara, tórax y abdomen persisten. La paciente evolucionó de manera favorable: el cuadro clínico mejoró (Figura 2), los valores de laboratorio se normalizaron y salió de alta con indicaciones de tratamiento tópico con cremas hidratantes y glucocorticoides en las zonas donde aún presentaba lesiones, así como prednisona por vía oral (con reducción gradual) y antihistamínicos condicionales a prurito (por vía oral). 


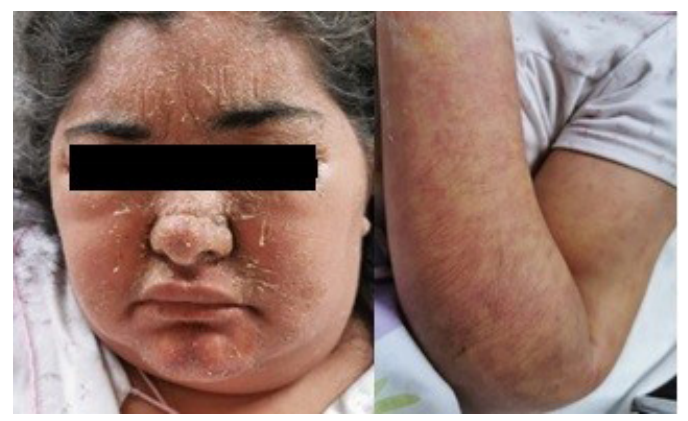

Figura 1. Edema facial y eritema en miembros superiores al cuarto día de hospitalización

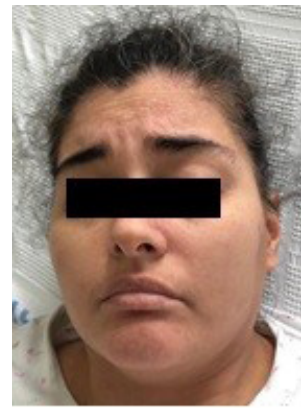

Figura 2. Paciente al momento del egreso hospitalario

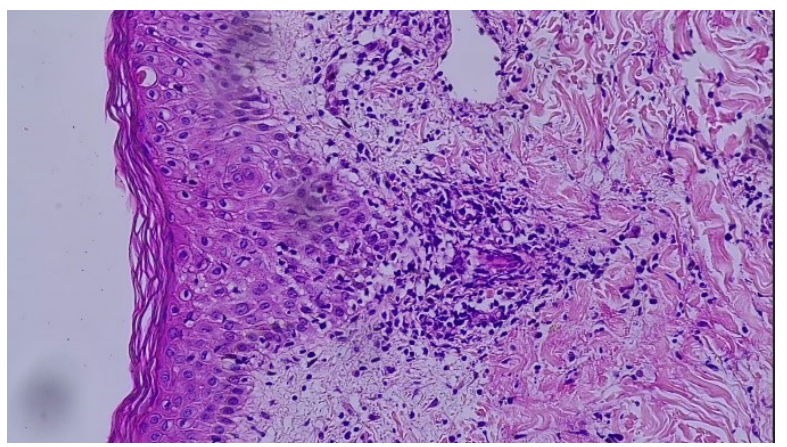

Figuras 3. Biopsia de piel: epidermis con disqueratosis, espongiosis, hipogranulosis, vacuolización de queratinocitos basales y exocitosis linfocítica (coloración hematoxilina-eosina)

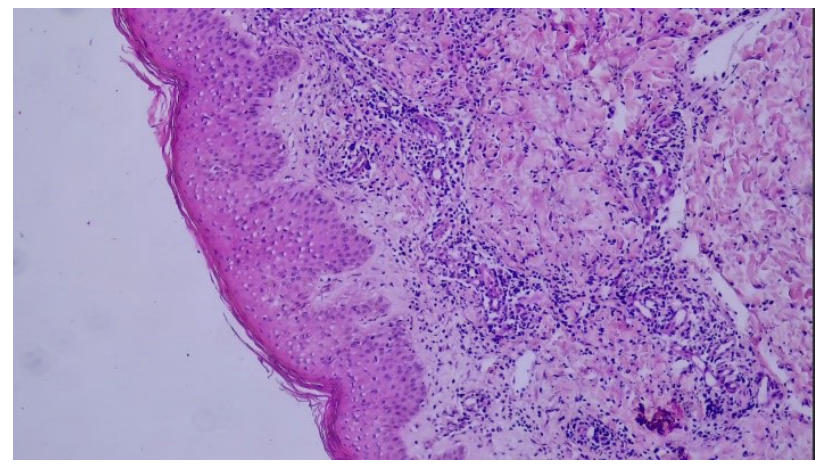

Figuras 4. Biopsia de piel: epidermis con infiltrado inflamatorio linfocítico, neutrofílico y eosinofílico (escaso) en dermis papilar perivascular e intersticial (coloración hematoxilina-eosina) 
Tabla 1. Exámenes auxiliares

\begin{tabular}{lccc}
$\begin{array}{c}\text { Exámenes } \\
\text { auxiliares }\end{array}$ & Al ingreso & Al quinto día & $\begin{array}{c}\text { A las cuatro } \\
\text { semanas }\end{array}$ \\
\hline Leucocitos & $6290 \mathrm{ul}$ & $26500 \mathrm{ul}$ & $10240 \mathrm{ul}$ \\
\hline Eosinófilos & $1 \%$ & $5 \%$ & $2,7 \%$ \\
\hline Fosfatasa alcalina & $528 \mathrm{UI} / \mathrm{l}$ & $1544 \mathrm{UI} / \mathrm{l}$ & $319 \mathrm{UI} / \mathrm{L}$ \\
\hline TGO & $160 \mathrm{UI} / \mathrm{l}$ & $26 \mathrm{UI} / \mathrm{l}$ & $23 \mathrm{UI} / \mathrm{l}$ \\
TGP & $176 \mathrm{UI} / \mathrm{l}$ & $69 \mathrm{UI} / \mathrm{l}$ & $26 \mathrm{UI} / \mathrm{l}$ \\
\hline
\end{tabular}

\section{DISCUSIÓN}

El síndrome de DRESS se caracteriza por un periodo de latencia prolongado desde la exposición al fármaco ${ }^{(7-13)}$ y una etapa de persistencia o empeoramiento del cuadro clínico luego de retirar el fármaco causante ${ }^{(2)}$. En la paciente, las manifestaciones clínicas aparecieron a las tres semanas de haber empezado el consumo de la carbamazepina y empeoraron, lo que fue evidente en los exámenes auxiliares tras cinco días de haber retirado el medicamento. Las manifestaciones clínicas fueron cutáneas y sistémicas. Las cutáneas varían desde una erupción maculopapular hasta lesiones urticariales, pústulas, vesículas, ampollas, púrpura, eritrodermia o liquenificación que abarcan más de la mitad de la superficie corporal. En la etapa de resolución se puede observar zonas de descamación ${ }^{(4,7)}$. Según Kardaun et al. ${ }^{(17)}$, el $76 \%$ de los pacientes presenta edema facial (característica distintiva de la enfermedad), que es evidente en las fotografías de esta paciente, así como las manifestaciones sistémicas que incluyen fiebre, adenopatías, alteraciones hematológicas y daño de órganos como el hígado, riñón, pulmón, corazón, entre otros, además de las manifestaciones hematológicas y el daño hepático agudo. Las manifestaciones hematológicas incluyen leucocitosis, eosinofilia y linfocitos atípicos ${ }^{(1,5-13)}$. El hígado es el órgano que está afectado con mayor frecuencia ${ }^{(8-13,18)}$, y esta lesión es una de las principales causas de muerte en los pacientes con síndrome de DRESS (1-6). El daño hepático se manifiesta con hepatomegalia o aumento de las enzimas hepáticas ${ }^{(8-13)}$ y con un patrón hepatocelular, colestásico o mixto ${ }^{(9)}$. En el estudio realizado por Lin et al. ${ }^{(18)}$, el patrón más frecuente fue el colestásico.

Por lo general, los hallazgos histopatológicos de reacción adversa a medicamentos son inespecíficos y variables ${ }^{(6,7)}$. En la mayoría de pacientes con DRESS se ha descrito un patrón caracterizado por infiltrado dermal superficial denso (más que en otras erupciones) que puede incluir granulomas, además, un infiltrado eosinofílico que no es siempre prominente, infiltrado perivascular o en banda, eritrocitos extravasados y edema dermal ${ }^{19}$. En la biopsia de piel se observa un infiltrado que puede estar compuesto solo por linfocitos (1/3 de casos), por linfocitos y eosinófilos (1/3), por linfocitos, neutrófilos y eosinófilos $(20 \%)$, linfocitos y neutrófilos (10\%), y solamente neutrófilos (5\% de casos) ${ }^{(20)}$
(Figuras 3 y 4). También se observan cambios epidérmicos como alteración vacuolar de la capa basal, espongiosis, paraqueratosis, apoptosis o formación de pústula subcorneal (19).

Debido al variable espectro clínico del síndrome de DRESS, se han planteado diversos criterios para su diagnóstico. Bocquet et al. (1) fueron los primeros en describirlo como el cuadro clínico que incluía erupción cutánea, anomalías hematológicas (eosinofilia o linfocitos atípicos) y afectación sistémica (adenopatías, elevación de transaminasas, carditis o nefritis). Posteriormente, tanto el Registro Europeo de Reacciones Adversas Cutáneas Severas (RegiSCAR) como el grupo de consenso japonés (J-SCAR) propusieron criterios diagnósticos. El primero clasifica el DRESS como posible, probable o definitivo ${ }^{(17)}$; mientras que el J-SCAR define este cuadro como típico (7 puntos) o atípico (5 puntos), e incluye la reactivación de VHH 6 como un criterio ${ }^{(21)}$. De acuerdo a estos parámetros, la paciente obtiene una puntuación de 5 , que corresponde a un síndrome DRESS probable (para RegiSCAR) y uno atípico (según J-SCAR).

El manejo de esta enfermedad se basa en su reconocimiento temprano y la suspensión inmediata del fármaco, como en este caso, en el que se suspendió la terapia con la carbamazepina de manera oportuna ${ }^{(7-12,22)}$; de esta manera se pudo haber limitado el daño hepático ${ }^{(10)}$. La fiebre y los síntomas cutáneos pueden ser aliviados con antipiréticos y glucocorticoides tópicos o antihistamínicos, respectivamente, estos medicamentos fueron administrados a la paciente ${ }^{(7,17)}$. Aunque el empleo de glucocorticoides sistémicos carece de una sólida evidencia que lo respalde, la paciente recibió prednisona ( $1 \mathrm{mg} / \mathrm{kg} /$ día), ya que puede disminuir los síntomas y los valores de los exámenes de laboratorio; sin embargo, se requiere una reducción progresiva de la dosis durante 3 meses, por lo menos, para evitar la recurrencia. Se debe considerar emplearlos por vía endovenosa en los pacientes que presentan daño de órganos grave o que no muestran mejoría con el tratamiento por vía oral (7-12,22,23). La paciente recibió corticoides endovenosos porque se evidenció un daño hepático. Otras terapias alternativas incluyen inmunoglobulina endovenosa, plasmaféresis o inmunosupresores como ciclosporina, ciclofosfamida e 
incluso fármacos antivirales que no consideramos utilizar ante la remisión del cuadro ${ }^{(7-12,22,23)}$.

Durante la hospitalización, las manifestaciones cutáneas y los valores de laboratorio deben ser monitoreados; sin embargo, la recuperación completa podría tardar semanas e incluso meses ${ }^{(7)}$. Además, es necesario considerar que estos pacientes pueden presentar secuelas a largo plazo como enfermedades autoinmunes, anemia crónica, falla renal, entre otras, por lo que requieren una vigilancia continua posterior a la salida del hospital ${ }^{(7-12)}$.

En el caso descrito, se consideró que el cuadro clínico estuvo relacionado con la carbamazepina de liberación prolongada, que fue el último fármaco agregado al tratamiento habitual. Si bien esta presentación se ha asociado a menos efectos adversos (16), en teoría la producción y acumulación de metabolitos secundarios del metabolismo de la carbamazepina serían la causa del cuadro clínico de la paciente. Tras seis meses de seguimiento, y sin haber recibido glucocorticoides por más de tres, la paciente no ha presentado un nuevo evento.

Contribución de los autores: Deborah Ximena Arzani Lezcano ha participado en la concepción, diseño y redacción del artículo, recolección de resultados, análisis, interpretación de datos y convocatoria de la paciente. Olga Mercedes Viviana Burgos García ha participado en el aporte de material de estudio y en la redacción y revisión crítica del artículo. Elman Rolando Gamarra Osorio ha aportado el material de estudio y ha participado en el diseño, redacción, revisión crítica y aprobación de la versión final del artículo.

Conflictos de interés: Los autores declaran no tener conflictos de intereses.

Fuentes de financiamiento: El artículo ha sido financiado por los autores.

\section{REFERENCIAS BIBLIOGRÁFICAS:}

1. Bocquet H, Bagot M, Roujeau JC. Drug-induced pseudolymphoma and drug hypersensitivity syndrome (Drug Rash with Eosinophilia and Systemic Symptoms: DRESS). Semin Cutan Med Surg. 1996; 15(4): 250-7.

2. Shear NH, Spielberg SP. Anticonvulsant hypersensitivity syndrome. In vitro assessment of risk. J Clinical Invest. 1988; 82(6): 1826-32.

3. Chiou CC, Yang LC, Hung SI, Chang YC, Kuo TT, Ho HC, et al. Clinicopathological features and prognosis of drug rash with eosinophilia and systemic symptoms: a study of 30 cases in Taiwan. J Eur Acad Dermatol Venereol. 2008; 22(9): 1044-9.

4. Chen YC, Chiu HC, Chu CY. Drug reaction with eosinophilia and systemic symptoms: a retrospective study of 60 cases. Arch Dermatol. 2010; 146(12): 1373-9.

5. Simonart T, Tas S. Management of drug rash with eosinophilia and systemic symptoms (DRESS Syndrome): an update. Dermatology. 2003; 206(4): 353-6.
6. Shiohara T, Inaoka M, Kano Y. Drug-induced hypersensitivity syndrome (DIHS): a reaction induced by a complex interplay among herpesviruses and antiviral and antidrug immune responses. Allergol Int. 2006; 55(1): 1-8.

7. Behera S, Das S, Xavier A, Selvarajan S. DRESS syndrome: a detailed insight. Hosp Pract (1995). 2018; 46(3): 152-62.

8. Shiohara T, Kano Y. Drug reaction with eosinophilia and systemic symptoms (DRESS): incidence, pathogenesis and management. Expert Opin Drug Saf. 2017; 16(2): 139-47.

9. Cho YT, Yang CW, Chu CY. Drug Reaction with Eosinophilia and Systemic Symptoms (DRESS): An Interplay among Drugs, Viruses, and Immune System. Int J Mol Sci. 2017; 18(6): 1243.

10. Criado P, Criado R, Avancini J, Santi C. Drug reaction with Eosinophilia and Systemic Symptoms (DRESS) / Drug-induced Hypersensitivity Syndrome (DIHS): a review of current concepts. An Bras Dermatol. 2012; 87(3): 435-49.

11. Martínez-Cabriales S, Rodríguez-Bolaños F, Shear NH. Drug reaction with Eosinophilia and Systemic Symptoms (DRESS): how far have we come?. Am J Clin Dermatol. 2019; 20(2): 217-36.

12. Howard MC. DRESS Syndrome: drug reaction with eosinophilia and systemic symptoms. Pediatr Emerg Care. 2017; 33(7): 499-504.

13. Cacoub P, Musette P, Descamps V, Meyer O, Speirs C, Finzi L, et al. The DRESS syndrome: a literature review. Am J Med. 2011; 124(7): 588-97.

14. Kano $Y$, Inaoka $M$, Sakuma $K$, Shiohara $T$. Virus reactivation and intravenous immunoglobulin (IVIG) therapy of drug-induced hypersensitivity syndrome. Toxicology. 2005; 209(2): 165-7.

15. McCormack M, Alfirevic A, Bourgeois S, Farrell J, Kasperavičiūtė D, Carrington M, et al. HLA-A*3101 and carbamazepine-induced hypersensitivity reactions in Europeans. N Engl J Med. 2011; 364(12): 1134-43.

16. Powell G, Saunders M, Marson AG. Immediate-release versus controlled-release carbamazepine in the treatment of epilepsy. Cochrane Database Syst Rev. 2016; 12(12): CD007124.

17. Kardaun SH, Sidoroff A, Valeyrie-Allanore L, Halevy S, Davidovici $B B$, Mockenhaupt $M$, et al. Variability in the clinical pattern of cutaneous side-effects of drugs with systemic symptoms: does a DRESS syndrome really exist?. Br J Dermatol. 2007; 155(2): 422-8.

18. Lin I-C, Yang H-C, Strong C, Yang C-W, Cho Y-T, Chen K-L. Liver injury in patients with DRESS: a clinical study of 72 cases. J Am Acad Dermatol. 2015; 72(6): 984-91.

19. Patterson J. Weedon's Skin Pathology. Fourth edition. USA: Elsevier; 2006.

20. Hall B, Cockerell C, Chisholm C, Jessup C, Vanderfriff T, Motaparthi $\mathrm{K}$, et al. Non-neoplastic Dermatopathology. 2nd ed. USA: Elsevier; 2007.

21. Shiohara T, lijima M, Ikezawa Z, Hashimoto K. The diagnosis of a DRESS syndrome has been sufficiently established on the basis of typical clinical features and viral reactivations. Br J Dermat. 2007; 156(5): 1083-4.

22. Husain Z, Reddy BY, Schwartz RA. DRESS syndrome: Part II. Management and therapeutics. J Am Acad Dermatol. 2013; 68(5): 709-20.

23. Descamps V, Ben Saïd B, Sassolas B, Truchetet F, Avenel-Audran M, Girardin P, et al. Management of Drug Reaction With Eosinophilia and Systemic Symptoms (DRESS). Ann Dermatol Venereol. 2010; 137(11): 703-8. 


\section{Correspondencia:}

Elman Rolando Gamarra Osorio

Dirección: Andre Lalande 132 Urbanización Puertas del Sol

Trujillo, Perú.

Teléfono: +51968914330

Correo electrónico: elman.gamarra.osorio@gmail.com

Recibido: 18 de setiembre de 2020

Evaluado: 17 de octubre de 2020

Aprobado: 02 de noviembre de 2020

(c) La revista. Publicado por Universidad de San Martín de Porres, Perú. (c) Br $_{\text {Br }}$ Licencia de Creative Commons Artículo en acceso abierto bajo términos de Licencia Creative Commons Atribución 4.0 Internacional. (http://creativecommons.org/licenses/by/4.0/)

\section{ORCID iDs}

Elman Rolando Gamarra Osorio (1) https://orcid.org/0000-0003-3707-5340

Deborah Ximena Arzani Lezcano 1 https: / / orcid.org/0000-0002-9795-2475

Olga Mercedes Viviana Burgos Garcia ำ https: / / orcid.org/0000-0002-3171-9064 